\title{
Glucocorticoids in rheumatoid arthritis: the picture is shaping up
}

\author{
Frank Buttgereit, ${ }^{1}$ Johannes W Bijlsma ${ }^{2}$
}

Despite the well-known efficacy of glucocorticoids (GCs), it is more common than exceptional that their known adverse event profile and co-morbidity implications elicit fierce debates when discussing their benefit-risk profile. These discussions usually come up, especially among GC 'supporters' and 'opponents', ${ }^{1}$ when trying to elaborate recommendations on how to use these drugs best in the treatment of rheumatic diseases such as rheumatoid arthritis (RA), ${ }^{2}$ polymyalgia rheumatica, ${ }^{3}$ giant cell arteritis, ${ }^{4}$ systemic lupus erythematodes, ${ }^{5} 6$ myositis $^{7}$ and even systemic sclerosis. ${ }^{7}$ For example, very divergent opinions were learnt during work on the European League Against Rheumatism (EULAR) recommendations (2013 update) for the management of RA. ${ }^{2}$ As a result, only 73\% (the lowest majority level of all recommendations) of the members approved the suggestion that GCs should only be used as bridging therapy for up to 6 months, ideally tapering them at earlier time points. Interestingly, when looking at the members voting against it, about half of them thought the statement was too weak, whereas the other half considered it to be too strong. ${ }^{2}$ Nevertheless, the level of agreement (strength of recommendation) was quite high (mean of 8.9) on final anonymous grading. It should also be noted that the group did not explicitly discuss the chronic use of GC in established RA.

Indeed, many patients and quite some physicians are still uncertain about the actual benefit:risk ratio of GCs. ${ }^{1}$ This uncertainty might prevent optimal treatment under conditions where GC treatment is known to be of added value. Fortunately, our view on the most optimal use of these drugs does slowly but constantly maturate. This primarily results from thorough

${ }^{1}$ Department of Rheumatology and Clinical

Immunology, Charité-University Medicine Berlin, Berlin, Germany

${ }^{2}$ Department of Rheumatology \& Clinical Immunology, University Medical Center Utrecht, Utrecht, The Netherlands

Correspondence to Professor Frank Buttgereit, Department of Rheumatology and Clinical Immunology, Charité - University Medicine Berlin, 10117 Berlin, Germany; frank.buttgereit@charite.de analyses of accumulated data in order to update recommendations, but there are also new and often qualitatively better data coming in to enrich our knowledge. A very good example of the latter is the work by Roubille et al. ${ }^{8}$ These authors report carefully collected 7-year data from a prospective multicentre observational cohort of patients with early arthritis (ESPOIR). Current gaps in knowledge were addressed by analysing the tolerability profile of GC use in early arthritis. As a result, a comprehensive data set now lies on the table to be judged by everyone.

There are two key findings. First, the analysis of data of 602 patients with RA demonstrates patients with (versus without) GC treatment to be those with the greater use of non-steroidal anti-inflammatory drugs and disease modifying antirheumatic drugs, more active disease and higher C-reactive protein and anti-citrullinated protein antibody levels. These data confirm that there may be a significant bias in form of confounding by indication when analysing GC effects. Roubille and colleagues properly addressed this problem by performing a weighted Cox proportional-hazards analysis, with the use of propensity score and inverse probability-of-treatment weighting, and including age, gender, history of hypertension and GC treatment. When doing so, the real-life tolerability outcomes did not show any significant difference between RA patients with and without GC treatment. The facts that most of the patients who took GCs started this therapy during the first 6 months and that they received $<5 \mathrm{mg}$ prednisone per day clearly support the good safety profile of low-dose GCs for early active RA. Second, the mean dosage during the entire follow-up was only $3.1 \pm 2.9 \mathrm{mg} /$ day which represents a very low-dose GC treatment. ${ }^{9}$ Therefore, it should be stressed that these safety data do not automatically lend support to higher doses.

\section{HOW CAN WE ACHIEVE A POSITIVE BENEFIT-RISK PROFILE WHEN USING GLUCOCORTICOIDS IN THE TREATMENT OF PATIENTS WITH RHEUMATOID ARTHRITIS?}

The real-world data obtained by the French group fit perfectly with results recently published. A multidisciplinary EULAR group of experts including patients with rheumatic diseases aimed to define conditions under which long-term (3-6 months or longer) GC therapy has an acceptably low level of harm. ${ }^{2}$ Following a thorough analysis for the four most worrisome GC adverse effects (on bone, hyperglycaemia/ diabetes mellitus, cardiovascular diseases and infections), the group concluded that the risks of long-term GC therapy are defined by drug-specific parameters (dose and duration) but at least as much by patient-specific characteristics (eg, age, gender, genetic pre-disposition, co-morbidities, co-medication and individual lifestyle) (figure 1). Although robust evidence on the risk of harm was often lacking, long-term dosages of $\leq 5 \mathrm{mg}$ prednisone equivalent per day can be considered to have an acceptably low level of harm. This appears to be true for the majority of patients with the exception of patients at high risk of cardiovascular disease who may require preventive measures. ${ }^{2}$

\section{THE UPDATED VIEW ON USING GLUCOCORTICOIDS IN THE TREATMENT OF RHEUMATOID ARTHRITIS}

The view of the 2013 update of RA recommendations was that both shortterm symptomatic and long-term structural effects of GCs should be used in the form of a bridging therapy. Therefore, these drugs should be administered as long as it takes for conventional synthetic disease-modifying antirheumatic drugs (DMARDs) to reach their maximum effect, but should then be tapered if clinically feasible. This view has been further evolved in the most recent update of these recommendations. ${ }^{10}$ The new wording-a compromise in order to accommodate most of the concerns and suggestions raised during the Task Force's debate-is as follows (recommendation 6): 'Shortterm glucocorticoids should be considered when initiating or changing conventional synthetic DMARDs, in different dose regimens and routes of administration, but should be tapered as rapidly as clinically feasible.' This wording does acknowledge existing differences in GC application in terms of dose regimen and routes, that is, there are several different regimens for oral use, intramuscular injection and intravenous pulse therapy. It is now also being stated more clearly that GCs should be given as bridging therapy together with conventional synthetic DMARDs, either as part of the initial strategy or subsequently if this has failed. In contrast, GCs are usually not needed as a bridging 
therapy when biological or targeted synthetic DMARDs are used. The reason is that these drugs typically have a rapid onset of action and the infection risks may be potentiated. ${ }^{11} 12$

It should be noted that the second part of this recommendation has been left unchanged stressing again that GCs should be gradually reduced and ultimately stopped, ideally within 3-6 months. ${ }^{10}$ The main reason for this is that the long-term use of GCs, especially at doses $>5 \mathrm{mg}$ /day, should be avoided because of the potential occurrence of adverse effects. ${ }^{1}$ In addition, the Task Force explicitly stated that the DMARD therapy may have to be considered a failure (and is, therefore, in need of optimisation) if GCs cannot be withdrawn within the time frame mentioned above.

In our opinion, chronic use of low-dose GCs can be considered a realistic option for some patients, based on the following observations:

1. Recent data show that a large proportion of patients with early RA do stay on GC therapy for $>6$ months, indicating an acceptable balance between efficacy and safety. For example, the data reported by Roubille et $\mathrm{al}^{8}$ show that $64 \%$ of RA patients entire follow-up. The mean duration of total GC treatment was $1057 \pm 876$ days, which is much longer than the received low-dose prednisone for the

recommended maximum period of 6 months. Data recently published on the routine care of early RA point were in the same direction. ${ }^{13}$ More than 1300 patients were followed for up to 2 years in an early arthritis cohort (CAPEA), and complete 2-year data were available for 669 patients with RA. Seventy-seven per cent of those patients were initially treated with GC at different starting doses (26\% <7.5 mg, 29\% 7.5-20 mg and $45 \% \geq 20 \mathrm{mg}$ of prednisolone per day). Of note, after 24 months, 47\% still were on GC.

2. Also in established RA, there are many patients obviously being more or less constantly treated with GC. For example, recently published German data from the National Database of the Collaborative Arthritis describe a total of 8084 patients with RA. Forty-eight per cent of these patients received a mean dose of $5 \mathrm{mg}$ prednisone equivalent per day, whereby $8.5 \%$ were treated with daily dosages $<5 \mathrm{mg}$, $37.7 \%$ with $5-7.5 \mathrm{mg}$ and $2.1 \%$ with $>7.5 \mathrm{mg}^{14}$

3. As outlined above, for many patients, a therapy with dosages $\leq 5 \mathrm{mg}$ prednisone equivalent for $>6$ months may have an acceptably low level of harm and will provide therapeutic effects on top of the DMARD therapy.

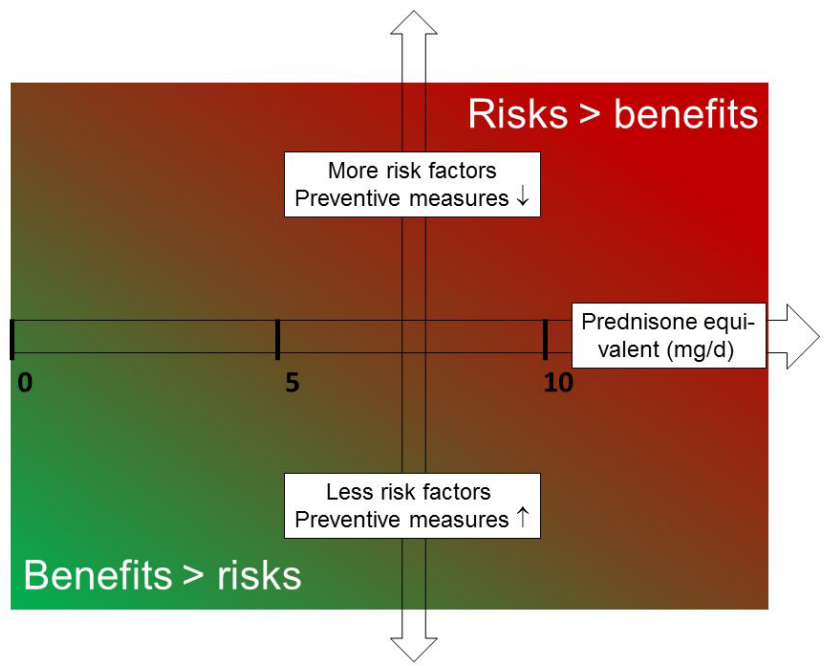

Figure 1 A matrix illustrating the benefit:risk ratio for a long-term therapy with GC. In general, lower GC doses together with the presence of less risk factors and more preventive measures lead to a lower level of harm, thereby leading to a better benefit:risk ratio of such a treatment (the green area). In contrast, higher GC doses together with the presence of more risk factors and less preventive measures lead to a higher level of harm, thereby leading to a worse benefit:risk ratio (the red area). More specifically, a therapy with dosages $\leq 5 \mathrm{mg}$ prednisone equivalent for $>6$ months may have an acceptably low level of harm in the majority of patients (with exception of patients at high risk of CVD who may require preventive measures). At dosages between $>5$ and $\leq 10 \mathrm{mg} /$ day, an acceptably low level of harm can only be assumed in the absence of certain risk factors and/or if appropriate preventive measures are taken. That is why the green colour gradually turns into red from bottom left to upper right. GC, glucocorticoids.
This assumption is backed by both the published EULAR Task Force work ${ }^{1}$ and the real-life data by Roubille et al. ${ }^{8}$

In conclusion, during treatment of both early and established RA, the risks of adverse effects induced by conventional GC can be minimised when following the established recommendations, ${ }^{15-17}$ and by considering each patient to be an individual person characterised by the presence or absence of certain risk factors and/or preventive measures. This will ultimately result in an adapted patient-specific therapy. Drawing updated conclusions based both on new data coming in and on balanced analyses of data already existing is a better way to go than relying on eminence-based statements (as has often been observed in the past and provided by both GC 'supporters' and 'opponents'). Not picking single observations or speculating on limited (and/or sometimes biased) data sets in order to convey certain negative or positive judgements on these drugs, but rather full and continuous analyses of the whole picture are what is needed. Glucocorticoids are (still) just too important to make half-hearted, opinion-driven statements.

Contributors Both authors have written together this article.

Competing interests FB has received consultancy fees, honoraria and travel expenses from Horizon Pharma, Mundipharma Int. Ltd. and Roche, and grant support from Mundipharma Int. Ltd. and Horizon Pharma. JWJB has received consultancy fees from Horizon, Mundipharma, Enceladus and SUN.

Provenance and peer review Commissioned; externally peer reviewed.

(C) Article author(s) (or their employer(s) unless otherwise stated in the text of the article) 2017. All rights reserved. No commercial use is permitted unless otherwise expressly granted.

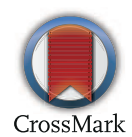

To cite Buttgereit F, Bijlsma JW. Ann Rheum Dis 2017;76:1785-1787

\section{S Linked}

http://dx.doi.org/10.1136/annrheumdis-2016210135

Ann Rheum Dis 2017;76:1785-1787.

doi:10.1136/annrheumdis-2017-211187

\section{REFERENCES}

1 Strehl C, Bijlsma JW, de Wit M, et al. Defining conditions where long-term glucocorticoid treatment has an acceptably low level of harm to facilitate implementation of existing recommendations: viewpoints from an EULAR task force. Ann Rheum Dis 2016:75:952-7. 
2 Smolen IS, Landewé R, Breedveld FC, et al. EULAR recommendations for the management of rheumatoid arthritis with synthetic and biological diseasemodifying antirheumatic drugs: 2013 update. Ann Rheum Dis 2014;73:492-509

3 Dejaco C, Singh YP, Perel P, et al. 2015 recommendations for the management of polymyalgia rheumatica: a European league against rheumatism/ American college of rheumatology collaborative initiative. Ann Rheum Dis 2015;74:1799-807.

4 Hoff P, Gaber T, Strehl C, et al. Immunological characterization of the early human fracture hematoma. Immunol Res 2016:64:1195-206.

5 Aringer M, Leuchten N, Fischer-Betz R. [Tapering and termination of immunosuppressive therapy: systemic lupus erythematosus]. $Z$ Rheumatol 2017;76:27-32.

6 Apostolopoulos D, Morand EF. It hasn't gone away: the problem of glucocorticoid use in lupus remains. Rheumatology 2016:kew406.

7 Postolova A, Chen JK, Chung L. Corticosteroids in myositis and scleroderma. Rheum Dis Clin North Am 2016;42:103-18.
8 Roubille C, Rincheval N, Dougados M, et al. Seven-year tolerability profile of glucocorticoids use in early rheumatoid arthritis: data from the ESPOIR cohort. Ann Rheum Dis 2017;76:1797-802.

9 Buttgereit F, da Silva JA, Boers M, et al. Standardised nomenclature for glucocorticoid dosages and glucocorticoid treatment regimens: current questions and tentative answers in rheumatology. Ann Rheum Dis 2002:61:718-22.

10 Smolen JS, Landewé R, Bij|sma J, et al. EULAR recommendations for the management of rheumatoid arthritis with synthetic and biological diseasemodifying antirheumatic drugs: 2016 update. Ann Rheum Dis 2017;76:960-77.

11 Listing J, Kekow J, Manger B, et al. Mortality in rheumatoid arthritis: the impact of disease activity, treatment with glucocorticoids, tnf $\alpha$ inhibitors and rituximab. Ann Rheum Dis 2015;74:415-21.

12 Lahiri M, Dixon WG. Risk of infection with biologic antirheumatic therapies in patients with rheumatoid arthritis. Best Pract Res Clin Rheumatol 2015:29:290-305.
13 Albrecht K, Callhoff J, Edelmann E, et al. [Clinical remission in rheumatoid arthritis. data from the early arthritis cohort study CAPEA]. Z Rheumatol 2016;75:90-6.

14 Albrecht $\mathrm{K}$, Huscher D, Eidner T, et al. [Medical treatment of rheumatoid arthritis in 2014 : current data from the german collaborative arthritis centers]. 2 Rheumatol 2017;76:50-7.

15 Palmowski Y, Buttgereit T, Dejaco C, et al. The "official view" on glucocorticoids in rheumatoid arthritis. A systematic review of international guidelines and consensus statements. Arthritis Care Res 2016.

16 van der Goes MC, Jacobs JW, Boers M, et al. Monitoring adverse events of low-dose glucocorticoid therapy: eular recommendations for clinical trials and daily practice. Ann Rheum Dis 2010;69:1913-9

17 Da Silva JA, Jacobs JW, Kirwan JR, et al. Safety of low dose glucocorticoid treatment in rheumatoid arthritis: published evidence and prospective trial data. Ann Rheum Dis 2006:65:285-93. 